\title{
Scheduling ON-OFF home control devices: Design issues and usability evaluation of four touchscreen interfaces
}

\author{
Catherine Plaisant \& Ben Shneiderman \\ Human-Computer Interaction Laboratory \\ Department of Computer Science \\ University of Maryland \\ College Park, MD 20742
}

\begin{abstract}
:
This article describes four different user interfaces supporting scheduling two state (ON/OFF) devices over time periods ranging from minutes to days. The touchscreen-based user interfaces including a digital, 12-hour clock, 24-hour linear and 24-hour dial prototypes are described and compared on a feature by feature basis. An informal usability test with 14 subjects, feedback from more than 30 reviewers, and the flexibility to add functions favors the 24-hour linear version.

* Address correspondence to Catherine Plaisant
\end{abstract}




\section{Introduction}

Contemporary homes include a large number of electronically controlled devices such as microwave ovens, VCRs, heating/cooling systems and security systems which can be scheduled to start and stop functioning at given times [TI89, SM88, JO89]. Typically each device has its own unique interface. As the number of devices in a home increases so does the mental load required to operate the devices since different procedures must be employed to operate each device.

Additionally, poorly designed user interfaces can make the scheduling task frustrating and difficult.

A solution to the problem of multiple user interfaces is to provide a home automation system consisting of a single user interface and the necessary hardware to control all the devices in the home using this interface. Such a user interface must then be general enough to support scheduling varied devices for a variety of tasks.

This research explores different mechanisms for scheduling two state (ON/OFF) devices to allow novice or occasional users to schedule events easily and correctly.

The use and representation of time is a strong component of western culture [ZE81] [MC47]. Our active life is cadenced by watches, personal calendars, and alarm clocks. Since one of our goals is to design user interfaces taking advantage of preexistent knowledge ("in the head"?) [NO88], it is important for a scheduling interface to make the best use of the common representation of the time: clocks, calendars, etc.

The scheduling of an event is also a data entry task in the sense that users have to enter values such as dates, times, number of hours etc. Data entry has been well studied [KA83, AN72], especially the entry of text from a keyboard, but also the use of dials and knobs which are more closely related to our watches. However, little can be found in the literature specifically related to the entry of temporal information. Weekly calendars have been used to ease the interpretation of the result of the scheduling of small group meetings [BE89]. Gould \& al. compared entry methods and selection methods for specifying dates [GO89]. The entry methods were found to be faster and more accurate than the selection methods but all methods used the keyboard only.

\section{Existing home control scheduling devices}

Simple scheduling devices can be found in stores or in our appliances (stoves, alarm clocks etc). They use either a dial (12 or 24 hours) or a digital display. When a dial is used, one turn of the dial is the maximum period usable (either 12 or 24 hours according to the dial). Two separate dials are sometime used to specify the start time and the duration or stop time. The precision is generally poor. Users either turn the whole dial, turn hands or turn a knob which turns the hands. Another common technique is to use pins on the circumference of the dial, which can be pushed or pulled to mark the ON period.

When a digital display is used, the precision is good, the number of functions is increased (it can give the current times, allows multiple alarms, etc.) but users are likely to need a manual to 
learn all the functions and retnetion may be poor. In some cases, the number of buttons is very limited to bring the cost or space down (e.g. on an alarm clock) therefore increasing the number of touches to perform (e.g up to 59 touch to change the minute value of an alarm with a single button) or the complexity of button combinations.

The VCR is the appliance which stands to benefit the most from improved scheduling interfaces since one key feature is its scheduled recording capabilities[NE89]. So far the VCRs scheduling interfaces are digital and use the numeric keypad and/or specialized buttons. Originally most VCRs used a small LCD bar to display the current time and also provide minimum feedback when a scheduling task was performed. The order of the data entry and the meaning of the numerous special buttons have to be learned. The editing of multiple events is difficult. Newer VCR's use the television screen during the interaction. The subtasks can be described: e.g. "Now type the channel number", and selections can be made from menus. Finally, another technique for the VCR is the use of bar codes. This technique is at its best when the user has access to a printed TV guide which associates a barcode with each program description (those guides are already used in Europe and Japan). A simple scan of the barcode schedules a program to be recorded. Instead of specifying days, times and channels, users only specify the program they want to record. The cognitive load associated with the scheduling task is greatly reduced. Unfortunately this technique is difficult to generalize to other devices: only a generic calendar can be used and dates and times still have to be specified.

In the industrial or management world, the word "scheduling" carries a far more complex meaning and is mainly associated with scheduling techniques (like CPM, PERT etc.). Computers are heavily used to apply those techniques and automatically plan the scheduling of construction, transportation, schools classes and use of rooms, etc. Here dates and times are calculated. Only in the editing phase are the user interface problems related to this research. Nevertheless it is interesting to note that even the earliest graphical scheduling approaches were based on a linear time-scaled representation [OB69].

\section{Design Issues}

After presenting the functional requirements and the hardware configuration used, this paper identifies issues associated with user interfaces for device scheduling. Four alternative touchscreen-based user interfaces are described. We then report the findings from a usability test conducted on the three most innovative prototypes. Our conclusions are also based on the comments of many informal reviewers (more than thirty) and on the feature by feature discussion of the interfaces.

The project had a highly practical orientation since this work was conducted in collaboration with an advanced technology company (Custom Command Systems Inc.). Custom Command designs and installs home automation systems which integrate entertainment, security, lighting, climate control, communication and other devices in affluent homes and offices. The company provided the requirements for the user interface and the hardware, and made comments about the 
designs.

\subsection{Functional limitations}

The functional requirements for the three user interfaces are identical. A subset of basic scheduling tasks was identified. These standard tasks fall into four general classes:

(1) single action (Turn OFF water at 9PM);

(2) one day event with both an $\mathrm{ON}$ and an OFF time.

(Turn the VCR ON at 9:30PM and OFF at 10:30PM on the same day);

(3) two day event with in a 24-hour time period.

(Turn the lights ON a 7PM and OFF at 1AM the next day);

(4) multi day event.

(Turn the alarm ON at 10:00AM on the 21 st and OFF at 6AM on the $30^{\text {th }}$ ).

For simplicity scheduling is limited to a single device at a time.

An event can occur on any day so the user must be able to select the day, and set the on and off time using only touch interaction. Appropriate visual feedback must be provided, so that users can verify their schedules. Some editing must be supported to allow users to revise an incorrectly scheduled event.

Other issues include editing schedules, copying schedules, scheduling repeated events on different days or at regular intervals and scheduling devices that are more than binary.

\subsection{Technical requirements}

The external hardware component of the device scheduling user interface consists of a touchscreen. The color, graphical screen displays are implemented under MS-DOS in the high resolution EGA mode (640x350 pixels). The sound generated by the small speaker of the CPU was also used by one of the interfaces. The home owner sees the screen as a fixture which is almost flush mounted into a wall. The touchscreen and all the home devices are controlled by an AT class personal computer.

Touchscreens were selected as the hardware side of the user interface because the expected users are not necessarily familiar with keyboards, mice, or other more complex hardware interface devices. In addition to providing a natural method of interaction, touchscreens are easy to learn, efficient, have no moving parts, require less work space than other input devices, and evoke a high degree of user satisfaction [PO88a] [PO88b].

The Microtouch touchscreen used returns a continuous flow of coordinates with a $1024 \times 1024$ resolution. This allows the dragging of objects and the use of a lift-off strategy for selection: when the finger touches the screen a cursor indicates precicely the exact coordinates read by the touchscreen. Only the lift off of the finger activates "hot" keys or sets an object being dragged. This strategy reduces the error rates and allows the selection of small targets (as small as one pixel [SE89]) 


\subsection{Description of the four user interfaces}

The four interfaces described here do not exhaust the possibilities but raise the issues involved in the design of such interfaces. Only the central components of the interfaces are described, descriptions of buttons used to return to the operating system or to reset or clear the interface are not considered central to the discussion here.

The schedulers are centered around two objects, a calendar and a scheduling clock mechanism. The clock mechanisms are the most distinctive:

- a digital style day and time setting device

- two analog 12-hour clocks

- a 24-hour dial

- and a 24-hour line.

\section{Digital scheduler}

The digital scheduler (Figure 1) represents the day and time digitally. The date and ON time are displayed on the top part of the screen. The OFF time is displayed on the bottom part of the screen. Arrows are displayed under and above each digit and the month names.

Initially the screen appears with both $\mathrm{ON}$ and OFF set to the same default value (the defaults are the current time of day and the current date). The arrow shaped buttons under and above each digit allow users to decrement or increment the digit. Similarly the month can be changed, and the AM/PM value toggled. The NEXT EVENT button saves the event and then redisplays the starting screen. CANCEL cancels the event displayed on the screen. 
Figure 1: Digital scheduler

(C)1988 University of Maryland

Figure 2: 12-hour clock scheduler

12-hour clock scheduler 
The 12-hour clock scheduler is shown in Figure 2. The screen is divided into two identical regions. The left side of the screen is used to set an ON time (or START) and the right one to set a OFF time (or STOP). Each scheduler consists of a monthly calendar, a 12-hour analog clock with 2 hands (similar to an analog wrist watch), and an AM/PM toggle.

When starting, only the calendars and QUIT button are active. The clocks are grayed-out to indicate their inactive state. When a calendar is touched, a red rectangle is displayed around the day under the user's finger. Lift-off strategy is used to reduce the error rate (the day is selected only when the finger is released, allowing users to drag their fingers until the red rectangle highlights the day they want to select). The calendar displays the current month with adjoining buttons to access the previous and next months.

Once a day has been selected on either calendar, the corresponding clock becomes active (which is indicated by highlighting the clock and adding hands to the clock face.)

The hands are moved by touching the screen. Then two strategies can be employed to move a hand. In the first strategy users grab the hand they want to move by touching the hand with their finger, and then drag it around the clock face to the desired position. If both hands are close together the initial touch point determines which hand is selected. The hour hand is selected only when the initial touch is inside the innermost part of the clock face.

The second strategy consists of directly touching the position where the hand needs to be moved. The numbers displayed around the clock are placed so that if users touch the number "3" the hour hands jumps to 3 , and if users touch the number "45" the minute hand jumps to forty five.

A toggle is associated with each clock to set the AM or PM. When touched, the toggle changes color, and switches to the opposite value when released.

When designing this version our hope was to provide a simple and stable interface using a familiar time representation (the wrist watch) and making a clear on-screen separation of the two actions required to schedule an event (set the $\mathrm{ON}$ and set the OFF). Entering a new event is the only possible task. More complex functions (like editing old events) are handled by a separate utility not covered here, and do not add complexity to the scheduling.

\section{4-hour line scheduler}

The 24-hour line scheduler (Figure 3) consists of a monthly calendar, two lines representing 24-hour days, and various flags (e.g. ON flags and OFF flags).

Initially the two lines are grayed-out as inactive, and no flags are available. Users select a first day by touching it on the calendar (using the same mechanism as the previous scheduler). This first day appears on the top line. The line is now colored, the graphics suggest the different times of the day by showing a sun in the middle, the moon at night and the length of daylight. 
Figure 3: 24-hour line scheduler

(C) 1988 University of Maryland

(C) 1989 University of Maryland

Figure 4: 24-hour dial scheduler 
If necessary a second day can be displayed (e.g. to schedule some device over a night or over several days). If a third day is selected, the first is replaced and the latest two days touched are displayed always in chronological order.

Stacks of the available flags are displayed. To schedule an event, users grab a flag by touching a flag source, and then drag it toward the time line placing it at the desired time position. For example to set a ON time, as users touch the ON-flag stack, an ON-flag cursor appears just above their finger and can be dragged to any of the two lines. While the flag is sliding on the line, exact feedback of the time is displayed under the stack of flags.

When the finger is released, the flag is attached to the line. The periods where the device are $\mathrm{ON}$ are shown in red on the line. A flag already set on a line can be grabbed for adjustment, or removed by dragging it away from the line. When a day is chosen by touching the calendar, the flags previously attached on it are redisplayed and can be edited.

A complete record of the scheduled events is also visible on the calendar. Thin red lines appear on the days in which events have been scheduled. For example if a user places an ON flag on the 19th, and an OFF flag the 24th, the numbers from 19 to 24 will be underlined in red. A CANCEL LAST button is available to remove the last flag set. Any flag can be removed either by dragging it away from the line (originally a scissor could also be dragged on a flag to cut it.)

When designing this interface, the possible generalization to complex tasks was a major concern but some of us feared that the linear representation of the day might be confusing for potential users.

\section{4-hour dial scheduler}

The 24-hour dial scheduler (Figure 4) was designed and implemented by Jeffrey Mitchell. It has a main window with a large calendar and a QUIT button. When a day is touched, a day window appears, partially overlapping the calendar. A day is represented by a 24-hour dial with one hand. The day window includes ON, OFF, CONTINUE and DONE buttons.

When a day is selected by touching the screen the select sound is emitted, the day is highlighted, and the dial scheduling window appears. Scheduling is accomplished by moving the hand around the dial to the appropriate position and then pressing $\mathrm{ON}$ or OFF as desired.

To turn the device $\mathrm{ON}$ at a given time, users first set the hand at the desired position (exact feedback of the time is given in the top right corner of the window), then press the ON button. A green line (radius) is drawn to mark the ON time. To turn the device OFF: users set the hand then press OFF. A red line is drawn to mark the OFF time. The area of the dial in which the device is $\mathrm{ON}$ is shown with an green arc connecting the two radial lines.

When both times are set, the DONE button is used to return to the calendar window. If the event is longer than 24-hours, the ON time is scheduled and then CONTINUE is pressed. When CONTINUE is pressed the dial window disappears and the calendar window is again active. A 
second day is selected from the calendar. When the dial reappear only the OFF and DONE buttons are active. Users set the OFF time and press DONE to finish that multiple day schedule.

This 24-hour dial representation is at its best when scheduling a event starting one day and ending the following day within a single 24-hour period: for example a user can turn a device ON the $29^{\text {th }}$ at $9 \mathrm{PM}$ and then, on the same dial, move the hand to $1 \mathrm{AM}$ and press OFF. The device will be $\mathrm{ON}$ for 4 hours up to $1 \mathrm{AM}$ on the $30^{\text {th }}$.

The calendar gives overall feedback for the scheduled events by displaying small dials on scheduled days. Events are displayed on the small dials by filling in wedges of the dial with a color which contrasts with the calendar background.

When designing this interface the readability of the screens was a major goal, as well as providing a familiar directive style of interaction. Another goal was to have a large calendar and large clock, thus leading to the need for overlapping display.

\subsection{Comparison of the schedulers}

The user interfaces can be compared along a number of different dimensions, including: (1) date selection, (2) time selection, (3) number of required touch-actions (4) precison, (5) feedback, and (6) sequencing and interaction style.

\section{(1) Date Selection}

Two selection strategies are used in the four user interface designs.

The digital interface uses buttons to increase or decrease the date displayed in a textual manner. This corresponds to the common desk calendar. If the default values of the day and month correspond to the current day, no action is required to schedule an event for the current day. Only a few screen touches are necessary to schedule events in the near future. On the other hand the setting of a distant date can take up to 9 touches in a 3 months range. The use of the buttons does not require high-precision screen touch.

Selecting a date from a calendar is a more direct selection process. Users directly touch the desired day. The monthly representation of the time seems easier. Only single touch is necessary to select a day in the current month (and a maximum of three touches for a selection in a three month range). A weekly calendar could also be used, but the paging would become laborious to reach a remote date (up to 12 in a three month range). The current date can be marked, as well as some feedback about the events already scheduled. The top line of the calendar displays the day names (Monday, Tuesday, etc.) that could be used for weekly events, for example, if Friday is selected, the event scheduled will take effect every Friday.

Depending on the size of the calendar, more or less precision and care is required. In the two first versions the calendar is always displayed; therefore its size is limited - each day is represented

by a $1 / 2 \mathrm{~cm}^{2}$ square. It requires the use of lift-off strategy to allow users to smoothly select a day without errors. In the third version (overlapping 24-hour dial) the calendar can occupy the whole screen and either land-on or lift-off strategy can be employed. 
The digital and 12-hour clock versions present two calendars while other versions present only one. For schedules within a single day, displaying two calendars might be confusing because users have to select the same day twice, however for events that start in one month and finish in another, two calendars are probably preferable.

\section{(2) Time selection}

The user interfaces used four different representations of the time of day. A digital clock, an analog 12-hour clock, a 24-hour dial, and a 24-hour line.

In the Digital scheduler, a numeric time is presented. Touching buttons increments and decrements the values. By having buttons available for each digit, the maximum number of touches can be limited to fourteen touches to set a time. This representation is very simple, readable and precise.

On the other hand this display doesn't give any natural feedback of the duration of the event nor of the relation to the current period with the other ones already scheduled. It doesn't show the actual state of the device. A separate table is necessary to list the other events set for the same day, which means that editing or deleting events involves other mechanisms not discussed here. An $\mathrm{AM} / \mathrm{PM}$ toggle has to be used if the common $2 \times 12$ hours notation is used.

The three other representations of the time are analog. The 12-hour clock used in the second version is very attractive as a common time representation. It could be assumed that everyone using a scheduler knows how to read and set a time on this type of clock. Users quickly discover that the hands have to be turned to set the time, and the touchscreen interface offers all its power by allowing the user to turn the hands with its finger.

There are some difficulties with analog clocks. When the hands are close together users must learn how to "grab" one or the other. Also the two 12-hour clocks showing the ON and OFF time don't give a clear image of the event duration, especially when the event overlaps more than an AM or PM period. Here again an AM/PM toggle is necessary and a separate table has to be used to see the times of the other events scheduled.

In the 24-hour dial version, the dial is still an analog clock but with a single hand. The 24hour representation avoids the AM/PM toggle which users are likely to forget in the previous representation. Here the single hand is easy to grab because there is no more need to disambiguate between the two hands of a 12-hour clock. The length of the event can be shown easily by filling areas of the circle. Several events can be represented and therefore edited. Events can also be scheduled across two days or within any 24-hour period using only one dial.

The fourth representation of the time is a 24-hour line. The image used here is the one of an appointment agenda on which events are drawn as lines or boxes connecting the beginning to the end of an event. Here again no AM/PM toggle is necessary. A graphic representation of the day can help confirm the scale marked on the line. Ideally the length of daylight, the phase of the moon or even the weather forecast could be suggested graphically. The length of the event can easily be read and multiple events can be shown on the same line. Only an extremely large number of events would overcrowd the line and require supplementary tools such as a loop. In our prototype only 2 lines are displayed simultaneously, but it is quite possible to display a larger number of lines 
allowing more complex tasks ( for example tasks involving several devices, every day of the week, etc.) Another alternative would be an interface with only one line, which could be expanded, contracted or scrolled to show the desired period in time.

\section{(3) Number of required touch-actions}

The following table compares the type and number of touch-actions necessary to perform a scheduling event (within a single month) and be ready to set another event. "T" stands for a touch and release, "D" stands for drag (touch an object - drag it - release), (<=max) means several actions and the italic style marks actions performed only when the event covers several days.

$\begin{array}{lllll} & \text { Digital } & \text { 12-hour clock } & \text { 24-hour line } & \text { 24-hour dial } \\ \text { ON date } & (<=7) \mathrm{T} & \mathrm{T} & \mathrm{T} & \mathrm{T} \\ \text { ON time } & (<=14) \mathrm{T} & \mathrm{D} \text { (hour hand) } & \mathrm{D} \text { (ON flag) } & \begin{array}{l}\mathrm{D} \text { (the hand) } \\ \mathrm{T} \text { (ON button) }\end{array} \\ & & \mathrm{D} \text { (min. hand) } & & \\ \text { OFF date } & (<=7 \mathrm{~T}) & \mathrm{T} \text { (AM/PM) } & T & \begin{array}{l}\text { (Continue or New date) } \\ \text { OFF time }\end{array} \\ & (<=14) \mathrm{T} & \mathrm{D} \text { (hour hand) } & \mathrm{D} \text { (OFF flag) } & \begin{array}{l}\mathrm{D} \text { (drag the hand) } \\ \mathrm{T} \text { (OFF button) }\end{array} \\ \text { Ready } & \mathrm{D} \text { (min hand) } & & \mathrm{T} \text { (DONE) } \\ \text { Total: } & \mathrm{T} \text { (next event) } & \mathrm{T} \text { (nMt } \text { (nevent) } & & 7\end{array}$

This table shows that the digital version could lead to a very large number of touches (even when using of set of controls for each digit), unlike the 24-hour Line version which requires a very limited number of actions. Of course dragging an object is a more complex action than pressing a button and involves better hand-eye coordination. More research would be necessary to compare precisely the four solutions.

\section{(4) Precision}

The Digital scheduler allows the user to easily set a time to the minute. Even greater precision could be attained by adding more digits.

In the 12-hour clock, each degree of angle corresponds to 1/6 minute of time while using the minute hand (30 degrees for 5 minutes.) It is relatively easy to set a time to the minute.

In the 24-hour dial, since only one hand is used, more careful control is necessary. Each degree of angle corresponds to four minutes of time. It is relatively hard to set times to the minute.

For the 24-hour line which uses the whole width of the screen, one hour is represented by only about $1 \mathrm{~cm}$, and allows users to easily select a time among 10 minute intervals. Many devices do not require high precision in their scheduling (furnace, air conditioning, etc.) and 5 or even 10 minutes intervals might be quite sufficient. If more precision were necessary, the 24-hour dial and the line versions would need some additional mechanism to adjust the time. Of course this will require additional touch actions that did not get taken in account in the previous section. The line 
time scale can also be stretched in the period of the day where the device is likely to be used, and compressed in other periods.

\section{(5) Feedback}

All four interfaces provide feedback on the current schedule. Only the 24-hour line and the 24-hour dial interfaces provide schedule feedback for previously scheduled events. The large calendar associated with the 24-hour dial version displays circles partially shaded in dates on the calendar when events are scheduled. It is easy to spot the days where events have been scheduled, and the reading of the times on the circles is simple if readers remember that it is a 24 -hour representation and not 12 . The main problem with this representation is the discontinuity of time: e.g. an single event overlapping the 12 midnight time will be represented by 2 separate shaded slices. The line scheduler shows a line on the calendar on days when events were scheduled. The position of the beginning and end of each line is proportional to the time of start and stop. Because the calendar is fairly small, it can be difficult to estimate the duration of an event or even to see it if the event is short.

\section{(6) Sequencing and Interaction Style}

The digital version as well as the analog 12-hour clock use two independent blocks to set the $\mathrm{ON}$ and OFF day and the time. Users can set all values in any order they wish, the OFF time can be adjusted first before the ON time, dates can be set after setting the times. The clear on screen separation of the two blocks should help the user identify the orders specifying an event. On the other hand this structure is limited to the simple type of events chosen for our study, involving only ON and OFF states for a device. But some devices can have more than two states (e.g. ON, OFF, and AUTOMATIC for a pool cleaning system). Supplementary blocks will be necessary for extra states.

The 24-hour dial version uses a different approach. The order in which an event is set is fixed: first select a day, then place the hand, press ON or OFF, move the hand to the other time position, and press the OFF or ON button. Such a strict sequencing needs to be learned. The user is guided by the buttons which appear only when they can be used. Instructions on the screen or with a voice output might be useful to teach a new user or to remind an occasional user. Devices having more than two states can be handled by adding supplementary buttons. Short cuts can also be provided with special buttons like "ALL DAY" or "ON for 1 HOUR".

The 24-hour line version uses yet another interaction style. Flags representing orders are placed on the line representing a day. Orders can be given in any order (OFF first, ON second). A relatively complex manipulation is required to pick a flag, drag it to the line, adjust the time and release the finger. It is a continuous single action. The use of the touchscreen gives a very smooth feeling of dragging flags on a whiteboard. The same manipulation is used to schedule an event as to edit or delete previously scheduled events: dragging a flag away from the line removes it, sliding a flag along the line changes its associated time. This unique concept should be easy to 
remember. Here also special flags can be made available for special devices: one hour flag for the lawn watering system, automatic mode flag for the pool cleaning, party flag for the lighting system.

The 24-hour dial version uses the overlapping technique to display the dial. This technique allows the designer to display a very large calendar as well as a large 24-hour dial, thereby making the selections easier. It also makes it clear that the first task is the selection of a date since no clock is yet displayed. On the other hand users must indicate their need to go back to the calendar by touching either the DONE button or the calendar itself (without making a selection).

\section{Usability evaluation}

We conducted a formative usability evaluation with two strategies. Fourteen novice subjects were given a benchmark set of tasks and were videotaped using a thinking aloud approach. We also asked knowledgeable colleagues, graduate students, and visitors to our lab to review and comment on three of the designs. Because our primary goal was to explore innovative user interfaces the digital version was not considered in the usability evaluation. The use of digital representation is well known but can be tedious and difficult to set. Digital VCR scheduling is known to be confusing and frustrating [NE89].

The novice subjects were video taped while learning to use the interface and while performing the same set of scheduling tasks with the three different user interfaces. Subjects used the schedulers in random order with two or three subjects assigned to each of the six orders. When the subjects completed the tasks their comments and preferences were solicited about the three schedulers.

The usability test was conducted in two steps during the fall of 1988 and the spring of 1989. Among the 14 subjects ( 5 women and 9 men) involved in this test, five were recruited from a group of retired university employees who regularly volunteer to participate in such university studies. This older population was thought to be more representative of owners of home control systems than groups of students often recruited for such studies. The other subjects were part of the University and Custom Command Systems non technical staff with ages ranging from approximately 30 to 60 .

When the subjects arrived in the lab, they were invited to sit in front of a touch-screen which

was placed on a normal desk. The video equipment was placed behind them. An experimenter sat on the side, trained the subjects and read each task aloud. The experimenter checked that the subjects completed each task successfully, solicited comments and filled in a form as tasks were performed. Each subjects used the schedulers for a total of approximately 40 minutes. Typical tasks were: "Schedule an ON time of 8:15 PM and an OFF time of 11:40 PM on the 2nd" or "Schedule the device to turn on at 11:00 PM on the 5th and OFF at 1 AM the following day."

The goal of the test was to understand advantages and disadvantages of each aspect of the designs, to identify the difficulties users had while using the schedulers, and hopefully select the best design to be implemented by Custom Command Systems.

\section{Results}

We will first discuss the use of the calendars then present the observations made for each of 
the versions.

\section{About the calendars:}

As foreseen all users identified the calendars as calendars and used them properly. The 24hour dial version's larger calendar was appreciated as more readable. On the other hand this last version did not use the lift-off strategy to select a day (the initial touch triggered the date selection) and despite the larger size of a day square, errors occurred and users had to back up to the calendar to reenter the proper date. This didn't happen as much in the other versions, showing again the advantage of the lift-off strategy [PO88]. Approximately a third of the subjects and many informal reviewers made remarks about the ease and smoothness of selection of a date on the calendar. One subject had some temporary difficulties mastering the lift-off technique and was the only one having problems with the small targets (about $1 / 2 \mathrm{~cm}^{2}$ ) of the little calendar. Users also suggested the addition of a "today" button or a default positioning on the current date.

About the 12-hour clocks version :

- As foreseen, users identified the clocks properly and were not surprised to have to turn the hands of the clock. Two subjects even avoided turning hands backward (to avoid braking them?)

- Users tended to forget to set the AM/PM toggle properly; therefore scheduling the event at the wrong time!

- Several users (approximately a fifth of the subjects and informal reviewers) did not realize that they had to choose the same day again for the OFF time. Of course it would be possible to set the stop day default value equal to the $\mathrm{ON}$ day, but this might provoke some unwanted scheduling of OFF time: users may want to schedule an ON only and not notice that a OFF has been set for them.

- When the two hands are close together, it is difficult for most of the subjects to reliably select one or another. The rule "use the inside for the hour hand and the outside for the minute hand" is not quite natural enough and needs to be remembered. A larger clock would help but this problem will always remain.

- Initially users easily lost control of the hand when crossing the clock border. We improved this by keeping control of the hand, once the finger landed inside the clock, even if the finger slid outside of the clocks. This change clearly improved the interaction.

- The AM/PM toggle has been pushed as much as possible away from the clock to avoid unwanted touch (and movement) of the close-by minute hand while switching the toggle.

Nevertheless that type of real error occurred, sometimes unnoticed!

\section{About the 24-hour line version:}

- The linear representation did not surprise anyone. Many users ( 3 during the test as well as many reviewers) commented on all the information that could be displayed on the line (e.g. the current moon phase, the weather forecast, length of daylight etc.) and expressed positive opinions of that representation.

- The AM/PM distinction was well perceived. The presence of the sun in the middle of the 
line allowed users to recognize AM and PM correctly without any labels.

- The principle of dragging flags on a time line seems to be natural. No subjects were confused. Two subjects expressed their great satisfaction with manipulating the flags. Some slid the flags around the other objects (like the sun or the top line), or brought the flag back to the corresponding pile when removing it from the line.

- Initially a strong "gravity" effect was used to keep the flag on the line. This effect had been installed to enable greater precision (minutes) by pulling up or down once the flag was on the line. Users felt they were loosing the control of the flag which did not always closely follow their finger. This strategy also made it very difficult to jump to the bottom line. This strong gravity was quickly redesigned. The gravity pulling the flag to a line is now only active when the finger is released close to the line (within $1 / 2 \mathrm{~cm}$ ).

- The possibility of re-grabbing a flag on a line to move or remove it was installed immediately, to respond to the great demand. This improvement and the suppression of the gravity made an obvious difference in the way subjects naturally handled the flags.

- Many subjects (approximately a third) expressed their confusion about the presence of a second line. Most of the tasks required only one line and subjects were surprised to still see the timeline used for the previous task when they called a new day timeline. This confusion may be linked to the laboratory environment for which subject don't expect a task to have any permanent effect.

- Subjects complained that they had to look under the stack of flags to read the time corresponding to their current position on the line. It would probably be preferable to display the digital feedback near the flag being adjusted or even on its label.

\section{About the 24-hour dial version:}

- As discussed earlier the large size of the calendar was appreciated.

- The strict sequencing of the interaction had to be described repeatedly to subjects, who often seemed to be searching for their next step. But they said they would probably remember the sequence if they used the system enough.

- Many contradictory comments were given about the labeling of the buttons (DONE or CONTINUE or CALENDAR)

- Subjects were uncertain about how to cancel or modify an event.

- Several persons remarked that the clock could be made larger to give more precision, and more space could be left between the clock and the buttons.

- Before the test the clock was labelled with 2x12 hours, AM on the right PM on the left. Surprisingly many reviewers said they would greatly prefer the $24 \mathrm{~h}$ (military) time notation for its clarity. We then tried the 24-hour labelling for the test. A fourth of the subjects complained about it, some seemed to be calculating the number to reach, demonstrating that the 24-hour notation is not well known. Each notation clearly has its detractors and a scheduling system should offer both notations as options.

\section{Error occurrence:}

We only considered as errors the cases where subjects scheduled an event for an incorrect 
time of the day (no errors at all were made on the date). Errors occurred in the two 12-hour clocks version when the AM/PM was forgotten and in the 12-hour clock and 24-hour dial versions because the hands were moved accidentally in an attempt to select a button (like AM/PM, ON, OFF, DONE...) and stayed unnoticed.

Of course many tasks were performed imprecisely (e.g. 10:35 or 10:39 instead of 10:40).

\section{Precision issue:}

Subjects always felt they had performed the task correctly, but in fact subjects very often did not try to set the time exactly at the value asked in the scenario. For example, if asked to start a furnace at 5:10 they considered it correct to start at 5:15. On the other hand, once all bugs where fixed, all negative comments heard were related to the difficulty of precisely adjusting the time. Some users (and about a quarter of the subjects of the test) tried very hard to get the exact time (personal differences? or was it the only challenge left?). Unfortunately our prototypes were intended to test the basic concepts and the fine tuning mechanisms have not been refined. In the 24-hour line or 24-hour dial, only a rough tuning allows users to increment a value by touching it directly. Such a simple tuning obviously needs a way to decrement as well as increment the time value ( 2 separate buttons under the time display). Other techniques can also be workable, for example, a magnifying glass effect. Fortunately, real home control situations do not always require much precision and the few devices that do, will need a customized tuning method (a VCR to the minute, a microwave to the second).

\section{Evaluating the quality of the feedback:}

Subjects were asked to perform tasks that were clearly independent from each others and there were no risks associated with a scheduling error. In a real situation, users of the scheduler are more likely to want to verify their work more carefully and also review it at a later time. This testing cannot give a complete assessment of the feedback quality. For example the 12-hour clock version does not allow the user to verify the events already scheduled but none of the subjects remarked about it. For the same reason no editing of the events was necessary here. We didn't get any specific comments about this issue.

\section{Overall rating of the prototypes by the subjects:}

Despite the small number of subjects, a wide range of personal preferences was observed. Each version was rated highest by some subjects. After each task we asked the subjects to grade the difficulty of the task on a 1 to 10 scale (1 being easy and 10 being hard). We also asked the subjects which version they would choose if a similar system were installed tomorrow in their house. The table below gives the values and results.

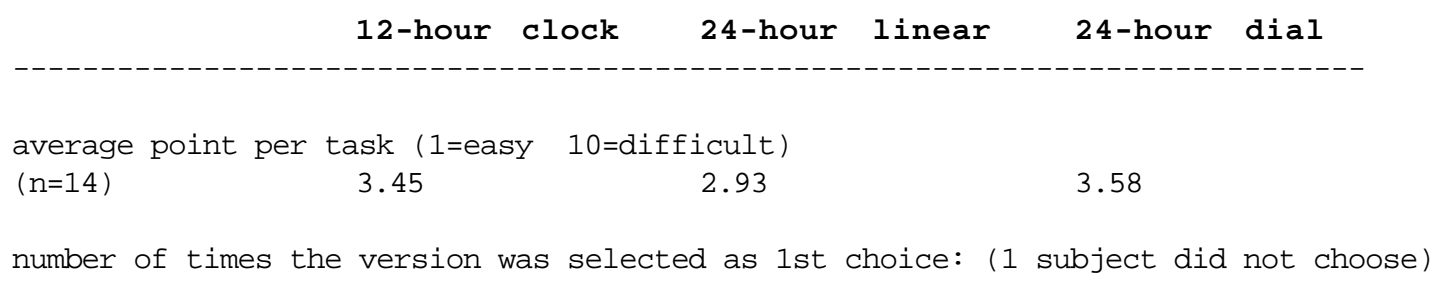


Those numbers correspond to overall ratings. We see that each version had its defenders. Our observations also showed that none of the versions was completely rejected. In this test, the precision attained seemed to be an important criteria in the overall grading of the 3 versions. We also saw that subjects did not always prefer (select as their first choice) the version that they rated as easier.

No significant conclusion can be drawn from this table and our final recommendations to Custom Command System were also based on the comments of additional reviewers.

\section{Other aspects:}

When asked, none of the subjects expressed any negative judgment against the general screen designs and colors. Nevertheless we know that the choice of the right color is again a challenge. What is the best color for ON? We also feel that the screen of the 12-hour clock version is too crowded with its 2 calendars, 2 dials and 2 toggles, that the calendars should preferably be moved to the top left corner of the screen and that, in general, every object be made larger.

\section{Discussion}

\section{About the use of the touchscreen:}

Most of the touch-screen applications encountered today still use large "button like" selectable areas. The development of our three schedulers was an opportunity to explore new touchscreen strategies with increased use of dragging or sliding movements. Dragging objects feels better than pressing buttons on a touchscreen. Turning the hands of a watch or sliding a flag provides a very nice feeling to the user: it is one more step towards direct manipulation. It would be interesting to compare the ease of controlling rotating or sliding objects with a touchscreen and with a mouse.

\section{About the schedulers:}

The 12-hour clock was initially implemented assuming that no one would have difficulty understanding how to use it. Because it has 2 hands it is more precise (selecting a minute is possible). Nevertheless we observed that it it's use is not as easy as we hoped. The rule allowing users to select a hand when both hands are close together is not obvious and the limited size of the clock bring more difficulties (a very large clock may solve the problem of desambiguation between hands). Another problem of a 12-hour clock is that the AM/PM is too easily forgotten.

A 24-hour representation (linear or circular) solves the double hands problem: only one mark is enough to specify a time, several events can be shown on the same day, the length of the event is readable. But the good precision of the 12-hour clock is lost and an additional tuning technique has to be used: a good stabilization of the touch-screen [SE89] or some tools like a zoom, + and buttons or slides, or a very large circle. The circle representation is well adapted to the scheduling of events happening during the night and overlapping 2 days but the lines would easily allow a larger number of days or devices to be represented on the same screen, making it a good candidate 
for complex tasks.

Another important issue is the interaction style. In the 24-hour dial version the chosen sequencing of action is fixed and has to be learned (first set the hand, then press ON, etc.). Comparatively the manipulation of the flag on the line is a single, smooth and easy to remember action. The line version also includes the editing and cancelling of previous events at no increase in the interface complexity (unlike the 12-hour or 24-hour dial versions which would need additionnal developments to include these possibilities (with a log book or additionnal buttons).

The use of overlapping objects allows larger objects, therefore more visible and easier to select. It also makes it easier to understand what has to be done when only a calendar is visible. On the other hand, when an error has been made (e.g. selecting the wrong date) is it harder to correct because you have to remove the overlapping objects. No general recommendation emerge from the litterature but we favor the non overlapping designs.

\section{Conclusions}

Each of these versions has its strengths and weaknesses. The usability test showed that each of these versions can be easily used. Contrary to our initial expectations the 12-hour clock version does not seem easier to use and is the only version where serious errors occurred. The limited number of subjects in the usability test does not produce statistically significant results but our global experience with the three interfaces during the test and with the numerous other users of the prototypes makes us believe that scheduler using lines and flags is a promising direction. It uses a simple and understandable concept and requires few touch-actions. It also has the flexibility for additional functions like the scheduling of more complex devices having more than two states, multiple day display, editing, etc.

It is clear that complex and novel graphical user interfaces require substantial usability testing in order to really assess which factors are best for a given task. It is also apparent that the comparison of the user interfaces serves less to select a single interface than it does to highlight the differences and suggest further improvements.

Only simple scheduling tasks have been considered here. This work is also a platform for the study of more complex tasks like the scheduling of several devices simultaneously, repetitive events, creation and scheduling of macro-events etc. Beside being useful in a home control system, easy to use scheduling interfaces could also find applications in professional environments like an audio-video room control or industrial process control.

\section{References:}

[AN72] Sponsored by Joint Army-Navy-Air Force Steering Comittee, Human Engineering Guide to Equipment Design. John Wiley \& Sons, 1972.

[BE89] Beard, D., Humm, A., A visual calendar for scheduling small group meetings. Technical report, Department of Computer Sciences, Univ. of North Carolina at Chapel Hill, 1989. 
[GO89] Gould, J., Boies, S., Meluson, A., Rasamny, M., and Vosburgh, A., Entry and selection methods for specifying dates, Human Factors, 1989, 31(2), 199-214.

[JO89] Johnson W., Command decision, Twentyone, VII,1, Jan-Feb.1989.

[KA83] Kantowitz, B. H., Sorkin, R., Human Factors: Understanding People-System Relationships, John Wiley \& Sons, 1983.

[MC47] Mac Carthy J. R., A Matter of Time - the Story of the Watch, Harper \& Brothers, 1947. [NE89] Nemy, E., New Yorkers, etc., The New York Times Sunday, September 24, 1989.

[NO88] Norman, D. A., The Psychology of Everyday Things, Basic Books, New York, 1988 [OB69] O'Brien, J. J., Scheduling Handbook, McGraw-Hill, 1969.

[PI86] Pickering, J.A., Touch-sensitive Screen: the Technologies and their Applications, International Journal of Man-Machine Studies, 25, 1986, 249-269.

[PO88a] Potter, R., Shneiderman, B., and Weldon, L., Improving the accuracy of touch-screens: an experimental evaluation of three strategies. Proceedings of the Conference on Human Factors in Computing Systems, ACM, New York, 1988, 27-30.

[PO88b] Potter R., Berman M., and Shneiderman B., An experimental evaluation of three touchscreen strategies within a hypertext database, International Journal of HumanComputer Interaction 1, 1, 1989, 41-52.

[SE89] Sears, A., Shneiderman, B., High Precision Touchscreens: Design Strategies and comparison with a mouse. Technical Report CAR-TR-450, CS-TR-2268, Human Computer Laboratory, University of Maryland, College Park, MD 20742.

[SH88] Sheer S., Input Devices, Academic Press, Inc., 1988.

[SM88] Smith R.L., Smart House - The Coming Revolution in Housing, GP Publishing, Inc., Columbia, Maryland, USA. 1988.

[TI89] Boosting Your Home's IQ, Time, January 23, 1989.

[ZE81] Zerubavel E., Hidden Rhythms : Schedules and Calendars in Social Life, The University of Chicago Press, Chicago, 1981.

Acknowledgments: Jeffrey Mitchell designed and implemented the 24-hour dial version and participated in the usability evaluation. We would also like to thank Jim Battaglia and Hank Levine from Custom Command Systems Inc. for their participation in the design of the interfaces described in this paper. We appreciate the support of the Maryland Industrial Partnerships program and Custom Command Systems Inc. in providing funding for this research. 